\title{
immersion - émersion
}

\author{
Thomas Leduc et Francis Miguet
}

Le Séminaire de Conception Architecturale Numérique (SCAN), devenu un des rendezvous de la communauté francophone de l'architecture, est une conférence qui rassemble des chercheurs autour de questionnements portant sur les implications du numérique dans la conception architecturale. Après avoir abordé les thèmes de l'esquisse numérique (2005), de l'image numérique (2007), des approches environnementales (2009), de l'espace collaboratif (2010), des complexités des modèles de l'architecture numérique (2012), de(s) l'interaction(s) des maquettes numériques (2014), du paramètre de modélisation morphologique au paramètre de spécification constructive (2016), cette $8^{\mathrm{e}}$ édition, organisée par l'UMR AAU à l'École Nationale Supérieure d'Architecture de Nantes les 25 et 26 octobre 2018, prolonge la réflexion en se donnant pour ambition de traiter de la dynamique immersion-émersion et de ses apports à la conception de projets architecturaux et urbains.

En accord avec les conclusions des précédentes éditions du séminaire, nous convenons que la conception architecturale numérique ne peut se résumer à la seule utilisation d'un ensemble d'outils logiciels et qu'elle transforme fondamentalement le processus de projet. L'injection de la mesure systématique comme celle du paramètre (au sens paramétrique) dans le continuum qui va du modèle à la matérialité, interagit avec l'ensemble des autres dimensions du projet, notamment historiques, culturelles, sociétales, esthétiques et économiques. Le processus de conception bénéficie alors d'apports potentiellement très diversifiés, tant en termes de réactivité et de capacité conversationnelle, que de réplicabilité et de transposabilité, mais aussi de prise en compte des éléments de contexte (l'insertion d'un nouveau bâtiment dans un environnement se doit notamment d'exploiter au mieux les ressources utiles de l'environnement tout en minimisant sa propre empreinte sur l'existant), du comportement énergétique, mécanique et thermique des structures, d'analyse du cycle de vie des composants, des modes d'habiter, etc.

Cette assistance à la conception, véritable levier ubique, multiforme et démultiplicateur, nous donne à voir des facettes et éléments de contexte qui nous étaient auparavant inaccessibles. Il nous immerge aussi - au risque de la submersion - dans le site, dans la maquette, dans les données, dans le projet... débauche de flux enveloppants, perpétuels quémandeurs d'attention.

SCAN'18 propose d'interroger la tension entre immersions multiples et émersion (et la notion d'attention qu'elle peut impliquer), en référence par exemple aux dispositifs numériques ubiques qui permettent au sujet in situ de disposer conjointement d'un point de vue ex situ, enveloppant, de réseaux. Cette question, ainsi que toutes celles qu'elle convoque, a de nombreuses implications pour la recherche et la pédagogie qui est conduite dans nos établissements et plus généralement pour la production architecturale et urbaine. 


\section{Une contribution plurielle}

À l'issue d'un processus de relecture en double aveugle, le Comité de Programme a décidé de sélectionner dix-huit contributions pour publication dans les actes de la conférence. Nous profitons de ce propos introductif pour remercier l'ensemble des auteurs qui ont soumis une proposition ainsi que tous les membres du comité de lecture qui ont accepté la tâche de relecture critique - indispensable à la qualité scientifique de cette édition.

Ces dix-huit contributions originales sont regroupées en cinq thématiques qui traitent toutes de questions relatives à l'immersion-émersion.

Les trois premières contributions présentent un ensemble de concepts et d'outils visant à l'amélioration de la pédagogie du projet au sein des écoles d'architecture.

En s'attachant plus particulièrement à la composition des décors des fenêtres tunisiennes, R. Barbouche déploie différentes méthodes d'analyse numérique dans le but de dégager des classes morphologiques, historiquement repérées. Mais au-delà de l'exemple, il s'agit avant tout pour l'auteur de «développer des outils d'aide à la conception dans le cadre de la pédagogie du projet et de l'informatisation du savoir-faire architectural ».

E. Bigot-Doll explore la question de l'échelle entre monde physique et environnement virtuel ; il s'agit « d'observer le développement de la créativité par hybridations successives d'outils numériques et analogiques au moment de la conception». Des miniatures physiques au monde virtuel, " la conception de projet à l'échelle architecturale immerge le concepteur dans des univers et interfaces fortement hétérogènes ».

Toujours en rapport avec la pédagogie du projet d'architecture, J. Milovanovic, D. Siret, G. Moreau et F. Miguet étudient l'influence de l'immersion dans un environnement virtuel sur l'augmentation des capacités d'apprentissage dans le cadre du studio de projet. Ils relèvent que l'utilisation de ces nouvelles modalités d'enseignement amplifient « le niveau d'engagement des étudiants dans le processus de conception », « réduit la difficulté de compréhension de l'espace » et « déplace la relation entre enseignant et étudiants ».

Les trois contributions qui suivent développent de nouveaux paradigmes, inspirés notamment du génie logiciel, afin d'améliorer la conception collaborative dans le processus de projet.

X. Calixte, S. Ben Rajeb et P. Leclercq s'interrogent sur les outils et méthodes utilisés dans les processus collaboratifs au sein des agences d'architecture. Ils proposent une méthodologie qui permet de "récupérer la trace des usages des outils des acteurs du projet ». Les auteurs décrivent les différents types de données encodées par les intervenants et discutent les premiers résultats obtenus dans un cadre pédagogique.

«Pendant trente ans l'architecture durable s'est concentrée sur les performances énergétiques des bâtiments et sur les économies d'énergie ». Ce constat conduit $\mathrm{C}$. Dautremont, C. Dagnelie et $\mathrm{S}$. Jancart à s'intéresser aux relations qui peuvent exister entre les nouveaux modèles économiques que sont le réemploi et l'économie circulaire dans 
l'architecture, et le processus BIM. Les auteurs mettent en parallèle la durabilité et le BIM et tentent d'établir « des ponts entre ces deux enjeux contemporains de l'architecture par la mise en lumière de freins et de leviers ».

En référence au paradigme de programmation par le flux du génie logiciel et par extension au domaine de la modélisation 3D, M. Yanatchkov propose «l'idée de modélisation par le flux comme un paradigme de modèle collaborant et de logiciel comme ressource ». Intrinsèquement donnée-centrée, cette approche se caractérise par une grande efficacité (calcul réparti), une pérennité accrue (maintenabilité des composants) et une forte évolutivité (réutilisabilité liée à la modularité).

Quatre articles mettent en évidence que s'il est un objet dynamique et transversal, le BIM n'en reste pas moins dans une phase transitoire, comme en atteste son appropriation difficile par les différents acteurs.

L'approche d'E. Cristia, P.-P. Zalio et F. Guéna met en résonnance maquette physique et maquette numérique, tout en dégageant leurs singularités ; si la maquette physique reste un objet statique essentiellement manipulée par l'architecte, la maquette BIM est un objet nouveau, immatériel, manipulé par l'ensemble des acteurs du projet, qui en traverse toutes les phases, de sorte que cette maquette tendrait à devenir le projet lui-même.

H.-J. Gless, D. Hanser et G. Halin interrogent les pratiques collaboratives dans le champ de la conception architecturale. Leur travail s'oriente plutôt vers "l'apport des sciences sociales et des pratiques orientées coordination comme vecteur de changement ». Les auteurs proposent, dans un cadre pédagogique, plusieurs expérimentations basées sur les méthodes agiles, et concluent qu'elles permettent « de mieux dessiner les contours d'une tâche de conception architecturale [...], ainsi que permettre une meilleure communication et cohésion de groupe ».

Après quelques précisions terminologiques et conceptuelles, E. Hochscheid et G. Halin nous invitent à une immersion dans les agences d'architecture afin d'identifier les facteurs d'influence et les stratégies d'évitement relatifs à l'adoption du BIM. Leur article se termine sur une forme d'avertissement, la rigidification de la réglementation et l'inertie au changement pourraient « impacter fortement la profession d'architecte et la structure des agences $»$.

Partant du constat que l'appropriation des outils de modélisation paramétrique en conception architecturale dans "les bureaux d'architecture de plus petite échelle » reste lacunaire, A. Stals, C. Elsen et S. Jancart nous immergent dans « l'analyse des résultats de deux formations [...] dispensées dans le cadre d'une formation continue sur le processus $\mathrm{BIM} » . \mathrm{Au}$ terme du processus, elles relèvent que « subsiste donc davantage le sentiment d'une complexité cognitive plutôt qu'une complète non-adéquation en regard de la pratique architecturale des petits bureaux d'architecture ».

Dans un autre registre, quatre articles posent la question de la perception des ambiances architecturales et urbaines et de leur transcription dans l'environnement numérique.

H. Belgacem, T. Leduc et M. Musy abordent la question du sentiment de confinement du piéton dans l'espace urbain, en prenant pour hypothèse que la densité vécue par l'usager n'est pas nécessairement en cohérence avec la densité réelle d'occupation du sol. Plusieurs 
indicateurs sont proposés et discutés ; leur implémentation numérique permet une analyse cartographique de l'espace urbain, exemplifiée sur une rue nantaise.

G. Giraldo, M. Servières, V. Tourre, V. Signorelli et A. Bonnet utilisent la vidéo pour caractériser les ambiances urbaines et tenter de dégager les paramètres qui influencent les modes d'occupation et d'appropriation d'un espace. La complémentarité de l'approche immersive et des simulations numériques est mise en évidence par la vidéo, celle-ci venant constituer un mode de restitution interactif des marqueurs d'ambiance.

Les ambiances sonores des espaces de performance sont abordées par A. Gross, qui propose un outil d'aide à la décision basé sur un modèle numérique de simulation acoustique. Les différents indices acoustiques, pour l'intelligibilité des voix et la perception musicale, sont explicités puis optimisés grâce à l'utilisation d'un algorithme génétique. L'outil développé est appliqué à l'optimisation formelle d'une coque acoustique.

L. Koubaa Turki, K. Raboudi et A. Ben Saci nous immergent dans la maquette numérique, au cœur de la parcelle. Partant du principe que l'enveloppe construite doit ménager l'accès au soleil du voisinage immédiat, ils proposent «de formaliser des stratégies de réglementation urbaine de prospect en vue d'une meilleure gouvernance de l'espace urbain à l'ère de la ville intelligente ».

Par une approche comparative des « codes de la perspective » orientale (chinoise) et occidentale, L. Lescop et Y. Lu nous immergent dans les prémisses d'une conception de visite virtuelle utilisant la perspective chinoise traditionnelle. Ils relèvent que «la transcription numérique de la perspective chinoise demande une approche phénoménologique pour laquelle les outils ne sont pas conçus » et que, par ailleurs, " reste également à définir un protocole de déplacement dans cet espace immersif à la chinoise ».

Enfin, trois contributions mettent l'accent sur les difficultés de passage du virtuel au réel. La confrontation à la matière induit de nouvelles problématiques : fabrication, montage, résistance, durabilité, etc.

Partant de travaux de la fin du XIXe siècle, C. Lefeu, M. Gillet et M. Mimram nous immergent dans des problématiques de fabrication de mécanismes paradoxaux, tout à la fois déployables mais aussi hyperstatiques. Ils nous confrontent, dans leur retour d'expérience, aux difficultés liées à la matérialité, au changement d'échelle, à la rigidité, à la nature des liaisons, etc.

P.-P. Pechenart, M. Gillet et M. Mimram nous immergent au plus près des surfaces planes, complexes voire à double courbures - nappes dont ils explorent la déformabilité en testant différents motifs de découpe. Cette analyse de sensibilité leur permet d'aboutir à la réalisation d'un pavillon formé d'une nappe de $40 \mathrm{~m}^{2}$ présentant de «nombreuses propriétés, à savoir une souplesse mêlée à sa résistance, une capacité de développement et de déformation, etc. ».

R. Zarcone, A. Hussein et M. Mansouri immergent le lecteur dans un ensemble d'expériences pédagogiques qui visent «à mener les étudiants à s'interroger sur la recherche de l'équilibre optimal entre les intentions architecturales [...] et les contraintes [...] d'un site réel dans une démarche de conception structurellement informée ». Cette morphogénèse « par le dessin, la statique graphique et la manipulation de maquettes, réelles et numériques » révèle, de la part des étudiants, " des difficultés de compréhension et donc d'anticipation de possibles problèmes liés à la fabrication et à l'assemblage ». 